\title{
Postnatal depression among Sudanese women: prevalence and validation of the Edinburgh Postnatal Depression Scale at 3 months postpartum
}

\author{
This article was published in the following Dove Press journal: \\ International Journal of Women's Health \\ 8 July 2015 \\ Number of times this article has been viewed
}

\author{
Dina Sami Khalifa ${ }^{1,2}$ \\ Kari Glavin ${ }^{3}$ \\ Espen Bjertness' \\ Lars Lien ${ }^{4,5}$ \\ 'Department of Community Medicine, \\ Institute of Health and Society, \\ Faculty of Medicine, University \\ of Oslo, Norway; ${ }^{2}$ Faculty of Health \\ Sciences, Ahfad University for Women, \\ Omdurman, Sudan; ${ }^{3}$ Diakonova \\ University College, Oslo, ${ }^{4}$ National \\ Advisory Board on Dual Diagnosis, \\ Innlandet Hospital Trust, Hamar, \\ ${ }^{5}$ Department of Public Health, \\ Hedmark University College, Elverum, \\ Norway
}

Correspondence: Dina Sami Khalifa Department of Community Medicine, Institute of Health and Society, Faculty of Medicine, University of Oslo, Postboks I I30, Blindern, 0318 Oslo, Norway Tel +249 9l 2364427

Email dinasami507I@hotmail.com
Purpose: Postnatal depression (PND) rates in low-resource countries have reached levels between $4.9 \%$ and 59\%. Maternal mental health has not been researched in Sudan, and there are no existing statistics on prevalence or significant risk factors for PND. Consequently, no screening test has been validated to screen for PND at the primary health care level. This study investigates the 3 months prevalence of PND and validates the Edinburgh Postnatal Depression Scale (EPDS) against the Mini-International Neuropsychiatric Interview (MINI).

Methodology: Pregnant Sudanese women in the second and third trimesters were recruited to the study during routine antenatal care visits in two major maternity hospitals in Khartoum state. They were screened for PND at 3 months postpartum using the EPDS. Test positive women were matched with test negative women according to nearest date of birth. A clinical psychologist verified their depression status using the MINI.

Results: The follow-up rate was $79 \%$. At a cutoff point of $\geq 12$, the 3 months prevalence of PND was $9.2 \%$. The sensitivity and specificity of the EPDS were $89 \%$ and $82 \%$, respectively. The EPDS and MINI showed a strong positive relationship (odds ratio =36). The positive predictive value and negative predictive value, using this study's prevalence, were $33 \%$ and $98.7 \%$, respectively. The receiver operator characteristic analysis showed an area under the curve of 0.89 . The cut-off point $\geq 12$ was the most acceptable point as it had the lowest number needed to diagnose (1.4) and a false-positive rate of $18 \%$.

Conclusion: The EPDS is a valid tool for screening for PND on a Sudanese population. It was accepted, easily administered, and understood by postnatal women. Health care personnel, especially village midwives, should be trained on screening and referral of depressed women for clinical evaluation and management. Due to limited resources available in Sudan, shorter screening tests need to be validated in the future.

Keywords: Edinburgh Postnatal Depression Scale, Sudan, Epidemiology, Sensitivity, specificity, developing countries

\section{Introduction}

Maternal mental ill health is not recognized as an important determinant of maternal and child morbidity and mortality in Sudan as well as other resource poor countries. ${ }^{1}$ There is a range of mental illness that women may experience during pregnancy and after delivery: antenatal and postpartum stress, anxiety, depression, and psychosis. ${ }^{2}$ Global research on maternal mental illness has shown strong evidence-linking maternal ill health with compromised child outcomes. Postnatal depression (PND) is a mental illness exhibiting the strongest link to adverse child outcomes; it is under diagnosed 
and under treated. ${ }^{3,4}$ According to the World Bank, 13\% of depression cases go into self-inflicted harm. ${ }^{1}$ PND has negative effects on the newborn's nutrition and breast feeding habits, ${ }^{5,6}$ cognitive development, ${ }^{7}$ and future mental health. ${ }^{8,9}$ The theory behind this association is that mental ill health of pregnant women disrupts the fetus's "well being" in utero as well as the mother-child relationship and daily child care practices after birth. ${ }^{10}$

PND has been thoroughly investigated in developed countries. Only recently has it been studied in women in low-resource countries. It has been postulated that women are "protected" from perinatal mental illness in such settings due to the influence of the social setup and practices that provide support for women during pregnancy and the postpartum period. ${ }^{11}$ Prevalence of PND in countries with poorer settings range from lower prevalence to almost double that in developed countries. There is around 5\% prevalence of PND among Nepalese women, ${ }^{12}$ 9\% among Mongolian women, ${ }^{13}$ $15 \%$ in Thailand, ${ }^{14}$ Brazil, ${ }^{15}$ and People's Republic of China ${ }^{16}$ while it is as high as 53\% in Guyana ${ }^{17}$ and $59 \%$ in India. ${ }^{18}$

There are no recent maternal mental health statistics available in Sudan. The most recent published studies on mental health in Sudan were four studies conducted in 2003, 2005, and 2009; three were conducted in households in camps for internally displaced people, ${ }^{19,20}$ and only one was conducted among young girls between the ages of 12 and $19 .{ }^{21}$ Eleven percentage of surveyed girls (1,107 total) reported symptoms of severe depression. To the best of our knowledge, maternal mental health has never been studied in Sudan, neither are there any national projected statistics for PND. It is postulated that prevalence of PND in Sudan is lower than average global figures due to the quality social support postpartum women receive in the puerperal period from women in their families and community. In addition, Sudanese culture encourages and incentivizes procreation; hence, women are at a higher social status if they were able to reproduce.

Edinburgh Postnatal Depression Scale (EPDS) was translated and validated in several countries and populations using different gold standard tests. In the Arab world, it was translated and validated in the United Arab Emirates by Ghubash et al in 1997..2 In an African setting, it was translated and validated in South Africa, ${ }^{23}$ Ethiopia, ${ }^{24}$ and Nigeria. ${ }^{25}$ Validation of screening instruments is important, as a valid screening instrument should measure what it claims to measure and should maximize detection of those who truly have the condition especially in low-resource settings. This study is part of a bigger follow-up study that determines risk factors for PND at 3 and 8 months postpartum. The aim of the present study is to measure 3 months prevalence of PND and to validate the EPDS in a Sudanese sample. Validation will determine the prospect of screening women for PND with the EPDS at the primary health care level.

\section{Methodology Study design}

In this cross-sectional study, women were recruited before delivery and then screened for PND at 3 months postpartum. Women presenting at two antenatal clinics (antenatal care [ANC]) in two major public tertiary hospitals were invited to participate in the study. The clinics provide routine ANC services for pregnant woman living within or outside the hospitals' catchment population. The hospitals were Omdurman Maternity Teaching Hospital (90\% of total sample) and Ibrahim Malik Teaching Hospital (10\% of sample). Omdurman Maternity hospital has one of the biggest catchment populations in Khartoum state. ${ }^{26}$ According to the hospital statistical reports, the hospital has 38,000 deliveries per year and 900 ANC visits per month, on average. Sudan follows the World Health Organization protocol for utilization of ANC services: pregnant women should receive a minimum of four visits during pregnancy. Khartoum state has the highest level of utilization of ANC services in Sudan and the highest level of institutional-based deliveries as well. ${ }^{27}$ ANC attendance in Khartoum state is $88 \%{ }^{27}$ This is the proportion of women that attend "at least one" ANC visit by a skilled provider during their pregnancy. Doctors provide $67 \%$ of these services in Khartoum state while village midwives and health visitors provide $21 \%$, mainly at primary health care facilities. About $11.7 \%$ do not receive any ANC during their pregnancy. ${ }^{27}$ Women from all localities of Khartoum state can access ANC services in Omdurman Maternity Hospital because access does not depend on location of residence. ${ }^{28}$ There is no linking between the different ANC clinics, so a woman could be registered at more than one clinic during her pregnancy.

Inclusion criteria were women of Sudanese nationality, in the second or third trimester, of any parity with full contact information (at least two working telephone numbers). Illiteracy was not an exclusion criterion as data collection was via interviews. The Study protocol was ethically approved in Sudan by the Sudan Ministry of Health and in Norway by REK (Regional Committees for Medical and Health Research Ethics, reference no 2013/353/REK). All women diagnosed as depressed by the Mini-International Neuropsychiatric Interview (MINI) were referred for adequate follow-up at local mental health clinics.

\section{Procedure}

Recruitment was intermittent during the period of April 2013-April 2014. Each clinic had three examination 
rooms and two doctors stationed in each. The women were randomly assigned to these rooms. The examining physician introduced the principal investigator to candidates immediately upon completion of their physical examination. More than 5,000 women attended the clinics during that period, the principle investigator approached 700 women, and 300 women gave consent for inclusion in the study. Participants were interviewed at recruitment (T0) and at 3 months post partum (T1).

\section{First interview (T0)}

In the first interview, basic demographic data and data on medical and obstetrical history, and history of violence and stressful conditions was collected. In addition, the interview assessed participants' satisfaction with a number of issues: marital support, sexual relationship, family and social support, living and housing conditions, income, self-satisfaction, and satisfaction with current quality of life.

\section{Second interview (TI)}

At 3 months postpartum, participants were interviewed on the history of current birth and puerperal period either through face-to-face interviews (at home or ANC clinic) or through phone interviews. Phone interviews were conducted to minimize loss of follow-up only when a woman was either away from Khartoum state or refused face-to-face interviews. The first EPDS screening was conducted at that time on 238 women of 300 . Test positive women were matched to test negative women based on "nearest date of birth." Only the tests positives and matched test negatives underwent validation by the MINI (gold standard test). The MINI was administered within 1 week of the EPDS screening by a clinical psychologist that was blind to the EPDS scores.

\section{Assessment instruments The EPDS}

The EPDS is a widely used, reliable, and validated screening tool for PND. It was specifically developed for screening at the primary health care level. ${ }^{29}$ The instrument has been translated and validated into 57 languages ${ }^{30}$ including Arabic and validated in 1997 in the United Arab Emirates. ${ }^{22}$ The tool has not been validated in a Sudanese population to the best of the author's knowledge. The EPDS screens for PND using 10 inventory questions investigating feelings occurring with the new mother within the previous 7 days. Each question has four possible answers rated from 0 to 3 . In this study, the EPDS was administered through a personal interview, and a test is "positive" for PND if the woman scores 12 or more out of 30 as set by Cox et al. ${ }^{29}$ The tool was originally designed to be self-administered, but studies have shown that the directed interview EPDS and self-completed EPDS are equivalent screening techniques for postpartum depression. ${ }^{31}$ According to Ghubash et $\mathrm{al}^{22}$ the EPDS has good internal consistency and reliability with a Cronbach's coefficient of 0.84 . In the current study, the Cronbach's coefficient is 0.83 , which is acceptable.

\section{The Mini-International Neuropsychiatric Interview}

This was the clinical evaluation gold standard test used to verify depression in EPDS test positives and matched test negatives. The MINI is a short structured diagnostic interview used by mental health professionals. It screens for major depressive symptoms in the previous two weeks prior to the interview. It has high sensitivity with good specificity and it is compatible with international diagnostic criteria of psychiatric disorders ICD- 10 and DSM- IV. ${ }^{32}$ The MINI is composed of closed ended questions set out in modules that evaluate 19 currently occurring psychiatric disorders. It has a family of subset interviews: the MINI plus (investigates 23 disorders), the MINI screen (used at the primary health care level) and the MINI Kid (for children and adolescents). The Arabic MINI used in this study was originally translated and validated to assess "major depressive symptoms" on a Sudanese population in two internally displaced person areas in Central Sudan. ${ }^{20}$

\section{Statistical analysis}

The validation part of the study was on a subsample of 40 women (20 EPDS test positives and 20 matched test negatives). SPSS was the statistical software used for data analysis. Prevalence of PND for the whole sample was calculated. Differences between test positives, and all the test negatives for selected variables were compared using crosstabulation. Validation indicators were calculated for the EPDS against the MINI at a cutoff point of $\geq 12$ : sensitivity, specificity, NPV, PPV, and the number needed to diagnose (NND). The receiver operator characteristic (ROC) curve and area under the curve for the EPDS were assessed to evaluate the most suitable cutoff point for screening for PND in the Sudanese population.

\section{Results}

A total of 238 women of 300 completed the EPDS at 3 months postpartum, resulting in a $79 \%$ follow-up rate. About 62 women were lost to follow-up at the time of screening due to personal refusal (4.7\%), husband refusal (4.3\%), and unreachable contact number $(11.7 \%)$. Women lost to 
Table I Distribution of variables among test positive women $(n=20)$ and "all" test negative women $(n=2 \mid 6)$

\begin{tabular}{lll}
\hline Variable & $\begin{array}{l}\text { No of test } \\
\text { positives }\end{array}$ & $\begin{array}{l}\text { No of test } \\
\text { negatives }\end{array}$ \\
\hline $\begin{array}{l}\text { Educational level } \\
\text { Postgraduate }\end{array}$ & 0 & 5 \\
University & 9 & 78 \\
Secondary & 5 & 62 \\
Primary & 6 & 66 \\
No education & 0 & 5 \\
Parity & & \\
Primigravida & 9 & 62 \\
Multipra (I-4) & 11 & 133 \\
Grandmultipra $(\geq 5)$ & 0 & 21 \\
Place of delivery & & 198 \\
Health facility & 20 & 18 \\
Home delivery & 0 & \\
\hline
\end{tabular}

Notes: Women are test positive for PND if they score $\geq 12$ in the EPDS. Women are test negative for PND if they score $<12$ in the EPDS.

Abbreviations: EPDS, Edinburgh Postnatal Depression Scale; PND, Postnatal Depression.

follow-up are not significantly different from women that completed the follow-up in age (27 years old and 28 years old, respectively), in parity (1.9 children and 1.8 children, respectively) or in educational level (Pearson chi square $P$-value $=0.705)$. At a cutoff point of $\geq 12$, the prevalence of PND at 3 months postpartum was $9.2 \%$; a total of 22 women scored $\geq 12$ in the EPDS, only 20 were included in the validation study.

\section{Sample characteristics}

The mean age is significantly different between test positive women and all test negative women, that is, depressed women are younger (24 years old and 29 years old, respectively, $P<0.001)$. The validation part of the study constitutes 20 EPDS test positives and 20 matched test negatives; two women who were test positive refused the MINI interview. The range of EPDS scores among test positives was 12-24 (mean score 15.4, SD 3.02), and the mean score of test negatives was 3.3,
SD 2.94) An overview of some demographics among test positives and "all" the test negatives are illustrated in Table 1.

\section{ROC analysis}

Validation of EPDS using the Mini as the "gold standard test" resulted in 18 women as "clinically depressed" and 22 as "not clinically depressed" (Table 2). The test characteristics at a cutoff point of $\geq 12$ were sensitivity $89 \%$ and specificity $82 \%$. The likelihood ratio (LR) for disease when the test is positive (LR+ve) is 4.9, and the LR of a negative test (LR-ve) is .134. There is a strong positive relationship between EPDS and MINI illustrated by an odds ratio of 36 . The PPV and NPV were both calculated using the Bayes' theorem incorporating the prevalence of PND calculated from this study, resulting in $33 \%$ and $98.7 \%$, respectively. The accuracy of EPDS for major depressive symptoms at each possible cutoff point is demonstrated in the ROC curve for the EPDS vs the MINI. The nonparametric analysis of the ROC yielded an area under the curve of $0.89(P<0.001$, $95 \%$ confidence interval 0.779-0.999; Figure 1 ). This indicates that the test can significantly distinguish between truly depressed and nondepressed women at the chosen cutoff point. The predictive values of EPDS at different cutoff points for major depression are illustrated in Table 3 . The cutoff points that have lower false-positive rates are $\geq 13, \geq 14$, and $\geq 15$. Although these points yield the least false-positive rates and higher PPV, the sensitivity of the test is compromised (72.2\%, 61.1\%, and 55.6\%, respectively) compared with a sensitivity of $88.9 \%$ at the chosen cutoff point of $\geq 12$. The NND is the lowest at a cutoff point of $\geq 12$ compared with cutoff points $\geq 10, \geq 11, \geq 13$, and $\geq 14$ (Table 3 ). The NND indicates the number of tests needed to gain a positive response on a diseased individual.

\section{Discussion and recommendations}

Estimates of prevalence of PND in lower income countries are quite variable, ranging from $4.9 \%{ }^{12}$ upto $59.4 \% .{ }^{33}$ The findings

Table 2 EPDS TI status vs MINI status ${ }^{\mathrm{a}}$

\begin{tabular}{llll}
\hline & MINI status & & Total \\
\cline { 2 - 3 } & Clinical depressed & Clinical not depressed & \\
\hline $\begin{array}{l}\text { EPDS TI status } \\
\text { Test positive } \\
\quad \text { Count }\end{array}$ & 16 & $40)$ \\
$\quad$ Within MINI status & $88.90 \% *$ & $18.20 \%$ & 20 \\
$\begin{array}{l}\text { Test negative } \\
\quad \text { Count }\end{array}$ & 2 & 18 & 20 \\
$\quad$ Within MINI status & $11.10 \%$ & $81.80 \% * *$ & \\
Total & 18 & 22 & 40 \\
$\quad$ Count & 18 & & \\
\hline
\end{tabular}

Note: ${ }^{\mathrm{T}}$ The kappa measure of agreement value $=.7$ ( $P$-value $\left.<0.00 \mathrm{I}\right)$. *Indicates sensitivity of the EPDS. **Indicates specificity of the EPDS.

Abbreviations: EPDS, Edinburgh Postnatal Depression Scale; MINI, Mini-International Neuropsychiatric Interview; vs, versus; TI, screening at 3 months postpartum. 


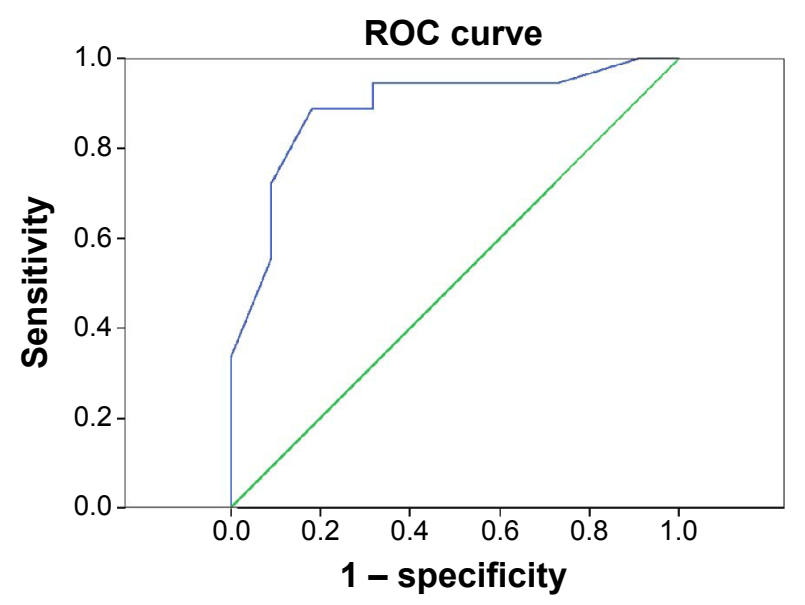

Figure I Receiver operating characteristic analysis of the EPDS total score against the Arabic MINI interview for DSM-IV diagnosis of postpartum major depression. Abbreviations: EPDS, Edinburgh Postnatal Depression Scale; MINI, MiniInternational Neuropsychiatric Interview; ROC, receiver operator characteristic.

from the present study show a prevalence estimate that is within the range of estimates from Arab and African countries. Arab Middle Eastern countries have estimates ranging from 16\% in United Arab Emirates, ${ }^{22} 19 \%$ in Morocco, ${ }^{34}$ and $21 \%$ in Lebanon. ${ }^{35}$ The present study estimate is comparable with estimates from Nigeria $10.6 \%{ }^{25}$ and Uganda $6.1 \% .^{36}$ Variation in estimates is due to variations in screening time and methodologies between the studies as well as to differences between populations in terms of economic and social circumstances, family relationships, and the reproductive and general health of women in each country. ${ }^{33}$

The study illustrates that screening for PND by EPDS on a local sample of 238 Sudanese women in an urban setting has good validity and will help in the identification of Sudanese women at risk for depression. The sensitivity and specificity of EPDS in the current study are similar to results retrieved from other validation studies that used similar cutoff points, for example, Cox et al, ${ }^{29}$ Berle et $a 1,{ }^{37}$ and Lawrie et al. ${ }^{23}$ The value of EPDS to health care providers depends not only on its sensitivity and specificity but also on the post-test probability of the disease after a positive or negative test, PPV, and NPV. The NPV in the current study was $98.8 \%$. Regmi et al, ${ }^{38}$ Teng et al, ${ }^{39}$ Aydin et $\mathrm{al}^{40}{ }^{40}$ and Lawrie et $\mathrm{al}^{23}$ detected similar high NPV: $100 \%, 99 \%, 94.5 \%$, and $92.2 \%$, respectively. In resource poor settings, the strength of the EPDS lies in its high NPV, meaning that with negative results we are confident that women are free from depression. Verification of the few false-positive cases by clinical interview is relatively inexpensive and noninvasive, especially that the NND in the current study is low.

Although the study estimated PND at 9.2\%, implementation of a screening program at any health care level will be most beneficial in detecting depressed mothers. This will not occur unless decision makers, especially in the mental health sector, understand the enormous public health burden depression has on the well-being of mothers and their newborns. Figures for women attending post natal care in Sudan are as low as $18 \% ;{ }^{27}$ hence, depressed women are easily missed. In this study, the EPDS was easy to administer; the questions were accepted and easily understood and did not revoke any hostile or undesirable reactions from women. Women who refused screening were more afraid of the stigma of being called "depressed." We recommend, as a first step in

Table 3 Range of EPDS cutoff scores and corresponding values of sensitivity, specificity, and predictive values in percentages ( $\mathrm{N}=40$ )

\begin{tabular}{|c|c|c|c|c|c|c|}
\hline Positive if equal or greater than & Sensitivity & Specificity & $\mathbf{P P V}^{\mathbf{a}}$ & NPV ${ }^{a}$ & I - Specificity (FPR) & $\overline{\text { NND }}$ \\
\hline$\geq 1$ & 100 & 9.1 & 10 & 100 & 90.9 & 11 \\
\hline$\geq 2$ & 94.4 & 27.3 & 11.6 & 98 & 72.7 & 4.6 \\
\hline$\geq 3$ & 94.4 & 36.4 & 13.1 & 98.5 & 63.6 & 3.2 \\
\hline$\geq 4$ & 94.4 & 40.9 & 13.9 & 98.6 & 59.1 & 2.8 \\
\hline$\geq 5$ & 94.4 & 45.5 & 14.9 & 98.8 & 54.5 & 2.5 \\
\hline$\geq 6$ & 94.4 & 50 & 16 & 98.9 & 50 & 2.3 \\
\hline$\geq 7$ & 94.4 & 50 & 16 & 98.9 & 50 & 2.3 \\
\hline$\geq 8$ & 94.4 & 59.1 & 18.9 & 99 & 40.9 & 1.9 \\
\hline$\geq 9$ & 94.4 & 68.2 & 23.1 & 99.2 & 31.8 & 1.6 \\
\hline$\geq 10$ & 88.9 & 68.2 & 22.1 & 98.4 & 31.8 & 1.8 \\
\hline$\geq 11$ & 88.9 & 77.3 & 28.6 & 98.7 & 22.7 & 1.5 \\
\hline$\geq 12$ & 88.9 & 81.8 & 33.3 & 98.8 & 18.2 & 1.4 \\
\hline$\geq 13$ & 72.2 & 90.9 & 44.6 & 97 & 9.1 & 1.6 \\
\hline$\geq 14$ & 61.1 & 90.9 & 40.5 & 95.9 & 9.1 & 1.9 \\
\hline$\geq 15$ & 55.6 & 90.9 & 38.2 & 95.3 & 9.1 & 2.2 \\
\hline
\end{tabular}

Note: at a prevalence of $9.2 \%$ for major depression. The bold indicates the psychometric properties (sensitivity, specificity, PPV, NPV, false positive rate and NND) of the EPDS compared to the MINI at a cut-off score $\geq 12$.

Abbreviations: EPDS, Edinburgh Postnatal Depression Scale; FPR, false-positive rate; NND, number needed to diagnose; NPV, negative predictive value; PPV, positive predictive value. 
addressing the issue, raising awareness on the importance and effects of maternal psychosocial disorders among the community and among all levels of health care providers to eliminate both patient and individual physician barriers. Acceptance by the community will make a screening program more effective. Raising awareness decreases the fear and stigma and allows for proper clinical management and follow-up through increased access to mental health services. Although there are no data on the effectiveness of routine screening in Sudan, we recommend training health personnel that work at the community level, especially nurse/village midwives, on using the EPDS package (screening and counselling) as they are usually the first point of contact for women at the postpartum period in most rural communities. They are well respected and trusted by community members, both men and women. They can enlighten their communities on the importance of this issue and may relieve some of the stigma associated with PND. This will gradually eliminate the patient barrier and will increase access to maternal mental health care. That type of cadre could also be trained to efficiently handle milder cases at the community level through counselling or social mobilization. Due to the lower educational level expected among women attending public health care, we also recommend administration of EPDS by personal interviews whether in a rural or urban setting. This necessitates additional training of personnel to be able to perform the screening in optimal conditions to avoid overestimated or underestimated responses. Clear guidelines on detection, referral and treating women with perinatal psychological morbidity should be part of routine maternity and postnatal care in Sudan. We recommend screening time beyond 6 weeks postpartum, since it is a social habit that postpartum women during the puerperal period receive immense social support from close family members. Depression is more likely to occur after that period. Referring all women suspected of depression for clinical evaluation and diagnosis is not feasible, especially in low-income countries. Referral should be for high-risk women detected by instruments validated on that population, setting, and disorder to minimize waste of limited resources. Hence, a cutoff point of $\geq 12$ is suitable for local screening for detecting truly depressed women due to low resources available and stigma associated with higher rates of false positives at lower cutoff points. Also, long-term larger-scale follow-up studies are needed in Sudan to evaluate the cost effectiveness of depression screening on the health of mothers and newborns. Evidence like that will assist in removing the organizational barriers to implementing depression screening among pregnant women in Sudan.
There is a lack of specific guidelines for the detection and treatment of perinatal depression in Middle Eastern and North African countries. Only two guidelines have been identified in the region through online search, the two are from Turkey. ${ }^{41}$ Until national guidelines are formulated, there are various valuable international guidelines that could be adapted to local settings. There is the new American College of Obstetricians and Gynecologist and the American Psychiatric Association guidelines for treating depression during pregnancy. ${ }^{42}$ There is also the National Institute for Care and Health Excellence guidelines: "Antenatal and postnatal mental health: clinical management and service guidance." It offers evidence-based advice on the recognition, assessment, care, and treatment of mental health problems in women during pregnancy and the postnatal period and in women who are planning a pregnancy. ${ }^{43}$ It is adaptable for implementation in Sudan and other resource poor settings either during routine ANC and postnatal care visits or during newborn immunization visits.

\section{Limitations and strengths}

There is lack of research in Sudan addressing maternal mental health, and this study was the first exploring PND. An important limitation to this study is that 300 women gave informed consent out of the 700 women approached. This puts the study at risk of bias that may have underestimated, or overestimated, the true prevalence of PND in Khartoum state. Another important limitation in the study design was not clinically interviewing the whole sample using the MINI. This was not feasible for the researcher. Having a clinical diagnosis for the whole sample would have set the validity of EPDS and the prevalence of PND across a range of cutoff points. One more limitation of this study was not including women attending private ANC services. Access to these women was limited and/or denied. Excluding these women may have affected the response rate and the estimates from this study. These limitations could be overcome in future studies. In addition, by recruiting women attending $\mathrm{ANC}$, we may have missed women who do not utilize these services. They could be more prone to PND. Women who do not attend ANC services in a hospital setting are more likely to have prenatal check-ups with midwives in their neighbourhoods. Village midwives could be a great asset in locating these types of women in future studies. The demographic features of this sample from Khartoum state are different from other states and regions in Sudan; hence, prevalence of PND across other states and ethnicities is not expected to be similar to this study's estimate since Sudan is a large diverse country and women have different social 
standings across different regions. Wide-scale screening for antenatal and PND conducted during, for example, household surveys could capture the true magnitude of the problem in Sudan. During such surveys, other types of maternal mental disorders can be explored as well. Choosing Omdurman Maternity Hospital for recruiting pregnant women to the study produced a sample that represents women in Khartoum state because it is the most utilized public maternity hospital in the state. Women from any locality in the state, rural and urban, are allowed to utilize its ANC services.

\section{Acknowledgments}

The authors are grateful to Miss Rania Adam and the clinical psychologist Ms Fatma Fazaa for their support and help during the follow-up phase in the data collection and clinical interviews. This study was supported in part by a grant from the Norwegian educational support fund.

\section{Disclosure}

The authors report no conflicts of interest in this work.

\section{References}

1. World Health Organization. Mental Health Aspects of Women's Reproductive Health: A Global Review of the Literature. Geneva: World Health Organization; 2009.

2. O'Hara MW, Wisner KL, Asher N, Asher H. Perinatal mental illness: definition, description and aetiology. Best Pract Res Clin Obstet Gynaecol. 2014;28:3-12.

3. Wachs TD, Black MM, Engle PL. Maternal depression: a global threat to children's health, development, and behavior and to human rights. Child Dev Perspect. 2009;3(1):51-59.

4. Engle PL. Maternal mental health: program and policy implications. Am J Clin Nutr. 2009;89(Suppl):963S-966S.

5. Harpham T, Huttly S, De Silva MJ, Abramsky T. Maternal mental health and child nutritional status in four developing countries. J Epidemiol Community Health. 2005;59:1060-1064.

6. Stuebe AM, Grewen K, Meltzer-Brody S. Association between maternal mood and oxytocin response to breastfeeding. $J$ Womens Health. 2013;22(4):352-360.

7. Davis EP, Glynn LM, Schetter CD, Hobel C, Chicz-Demet A, Sandman CA. Prenatal exposure to maternal depression and cortisol influences infant temperament. $J$ Am Acad Child Adolesc Psychiatry. 2007;46(6):737-746.

8. Weinstock M. The potential influence of maternal stress hormones on development and mental health of the offspring. Brain Behav Immun. 2005;19:296-308.

9. Murray L, Cooper PJ. The impact of postpartum depression on child development. Int Rev Psychiatry. 1996;8:55-63.

10. Chung EK, McCollum KF, Elo IT, Lee HJ, Culhane JF. Maternal depressive symptoms and infant health practices among low-income women. Pediatrics. 2004;113(6):e523-e529.

11. Stern G, Kruckman L. Multi-Disciplinary perspectives on post-partum depression: an anthropological critique. Soc Sci Med. 1983;17(15):1027-1041.

12. Ho-Yen SD, Bondevik GT, Eberhard-Gran M, Bjorvatin B. Factors associated with depressive symptoms among postnatal women in Nepal. Acta Obstet Gynecol. 2007;86:291-297.

13. Pollock JI, Manaseki-Holland S, Patel V. Depression in Mongolian women over the first 2 months after childbirth: prevalence and risk factors. $J$ Affect Disord. 2009;116:126-133.
14. Limlomwongse N, Liabsuetrakul T. Cohort study of depressive moods in Thai women during late pregnancy and 6-8 weeks of postpartum using the Edinburgh Postnatal Depression Scale (EPDS). Arch Womens Ment Health. 2006;9:131-138.

15. Faisal-Cury A, Tedesco JJA, Kahhale S, Menezes PR, Zugaib M. Postpartum depression: in relation to life events and patterns of coping. Arch Womens Ment Health. 2004; 7:123-131.

16. Wan EY, Moyer CA, Harlowa SD, Fan Z, Jie Y, Yang H. Postpartum depression and traditional postpartum care in China: role of Zuoyuezi. Int J Gynecol Obstet. 2009;104:209-213.

17. Affonsoa DD, Deb AK, Horowitzc JA, Mayberryd LJ. An international study exploring levels of postpartum depressive symptomatology. J Psychosom Res. 2000;49:207-216.

18. Nagpal J, Dhar RSG, Swati Sinha S, Bhargava V, Sachdeva A, Bhartia A. An exploratory study to evaluate the utility of an adapted Mother Generated Index (MGI) in assessment of postpartum quality of life in India. Health Qual Life Outcomes. 2008;6:107.

19. Federal Ministry of Health, Sudan. Sudan Mental Health; Draft National Policy. Khartoum: Federal Ministry of Health, Sudan; 2006.

20. Salah TTM, Abdelrahman A, Lien L, Eide AH, Martinez P, Hauff E. The mental health of internally displaced persons: an epidemiological study of adults in two settlements in Central Sudan. Int J Soc Psychiatry. 2013;59(8):782-788.

21. Shaaban KMA, Baasharb TA. A community study of depression in adolescent girls: prevalence and its relation to age. Med Princ Pract. 2003; 12:256-259.

22. Ghubash R, Abou-Saleh MT, Daradkeh TK. The validity of the Arabic Edinburgh Postnatal Depression Scale. Soc Psychiatry Psychiatr Epidemiol. 1997;32:474-476.

23. Lawrie TA, Hofmeyr GJ, De Jager M, Berk M. Validation of the Edinburgh Postnatal Depression Scale on a cohort of South African women. S Afr Med J. 1998;88(10):1340-1344.

24. Tesfaye M, Hanlon C, Wondimagegn D, Alem A. Detecting postnatal common mental disorders in Addis Ababa, Ethiopia: validation of the Edinburgh Postnatal Depression Scale and Kessler Scales. J Affect Disord. 2010;122:102-108.

25. Uwakwe R. Affective (depressive) morbidity in puerperal Nigerian women: validation of the Edinburgh Postnatal Depression Scale. Acta Psychiatr Scand. 2003;107:251-259.

26. Federal Ministry of Health, Sudan. Health Care Financing and Delivery. Khartoum: Federal Ministry of Health, Sudan; 2012.

27. Central Bureau of Statistics, National Ministry of Health. Sudan Household Survey 2010 - 2nd Round. Khartoum: Central Bureau of Statistics, National Ministry of Health; 2010.

28. Ibnouf AH, Borne HW, Maarse JA. Utilization of antenatal care services by Sudanese women in their reproductive age. Saudi Med J. 2007;28(5):737-743.

29. Cox JL, Holden JM, Sagovsky R. Detection of postnatal depression: development of the 10-item Edinburgh Postnatal Depression Scale. $\mathrm{Br}$ J Psychiatry. 1987;150:782-786.

30. Cox J, Holden J, Henshaw C. Perinatal Mental Health. The Edinburg Postnatal Depression Scale (EPDS) Manual. 2nd ed. London: RCPsych Publications; 2014.

31. Kaminsky LM, Carlo J, Muench MV, Nath C, Harrigan JT, Canterino J. Screening for postpartum depression with the Edinburgh Postnatal Depression Scale in an indigent population: does a directed interview improve detection rates compared with the standard selfcompleted questionnaire? J Matern Fetal Neonatal Med. 2008;21(5): 321-325.

32. Sheehan DV, Lecrubier Y, Sheehan KH, Amorim P, Janavas J, Weiller E. The MINI- International Neuropsychiatric Interview (M.I.N.I): the development and validation of a structured diagnostic psychiatric interview for DSM-IV and ICD-10. J Clin Psychiatry. 1998;59(suppl 20):22-33.

33. Fisher J, Cabral de Mello M, Patel V, et al. Prevalence and determinants of common perinatal mental disorders in women in low- and lowermiddle-income countries: a systematic review. Bull World Health Organ. 2012;90:139G-149G. 
34. Agoub M, Moussaoui D, Battas O. Prevalence of postpartum depression in a Moroccan sample. Arch Womens Ment Health. 2005;8:37-43.

35. Chaaya M, Campbell OMR, El Kak F, Shaar D, Harb H, Kaddour A. Postpartum depression: prevalence and determinants in Lebanon. Arch Womens Ment Health. 2002;5:65-72.

36. Nakku JEM, Nakasi G, Mirembe F. Postpartum major depression at six weeks in primary health care: prevalence and associated factors. Afr Health Sci. 2006;6(4):207-214.

37. Berle JO, Aarre TF, Mykletun A, Dahl AA, Holsten F. Screening for postnatal depression Validation of the Norwegian version of the Edinburgh Postnatal Depression Scale, and assessment of risk factors for postnatal depression. $J$ Affect Disord. 2003;76:151-156.

38. Regmi S, Sligl W, Carter D, Grut W, Seear M. A controlled study of postpartum depression among Nepalese women: validation of the Edinburgh Postpartum DepressionScale in Kathmandu. Trop Med Int Health. 2002;7(4):378-382.

39. Teng HW, Hsu CS, Shih SM, Lu ML, Pan JJ, Shen WW. Screening postpartum depression with the Taiwanese version of the Edinburgh Postnatal Depression Scale. Compr Psychiatry. 2005;46:261-265.
40. Aydin N, Inandi T, Yigit A, Hodoglugil NN. Validation of the Turkish version of the Edinburgh Postnatal Depression Scale among women within their first postpartum year. Soc Psychiatry Psychiatr Epidemiol. 2004;39:483-486.

41. Karamustafalıoğlu O. Major depressive disorder, mental health care, and the use of guidelines in the Middle East. J Clin Psychiatry. 2010; 71(suppl E1):e07.

42. Santoro K, Peabody H. NIHCM Foundation Issue Brief: Identifying and Treating Maternal Depression: Strategies and Considerations for Health Plans. Washington: National Institute for Health Care Management; 2010.

43. National Institute for Health and Care Excellence (NICE). Antenatal and Postnatal Mental Health: Clinical Management and Service Guidance. London: NICE; 2014.
International Journal of Women's Health

\section{Publish your work in this journal}

The International Journal of Women's Health is an international, peerreviewed open-access journal publishing original research, reports, editorials, reviews and commentaries on all aspects of women's healthcare including gynecology, obstetrics, and breast cancer. The manuscript management system is completely online and includes

\section{Dovepress}

a very quick and fair peer-review system, which is all easy to use. Visit http://www.dovepress.com/testimonials.php to read real quotes from published authors. 